\title{
Open reduction internal fixation of proximal humerus fractures
}

\author{
Marschall B. Berkes • Milton T. M. Little • Dean G. Lorich
}

Published online: 16 January 2013

(C) Springer Science+Business Media New York 2013

\begin{abstract}
The treatment of proximal humerus fractures continues to evolve. While the many of these injuries can be managed nonoperatively, a certain percentage require operative treatment. Open reduction internal fixation can offer excellent outcomes when performed in the appropriate patient and utilizing proper techniques. This article reviews the most up-to-date literature regarding all phases of proximal humerus fracture osteosynthesis, including diagnosis, imaging, anatomic considerations, surgical indications, fixation, and surgical outcomes.
\end{abstract}

Keywords Proximal humerus fracture - Neer classification · Open reduction internal fixation · Surgical indications . Proximal humerus blood supply $\cdot$ Locking plate $\cdot$ Proximal humerus complications $\cdot$ Avascular necrosis $\cdot$ Endosteal augment $\cdot$ Screw perforation $\cdot$ Fibula allograft

\section{Introduction}

The surgical treatment of proximal humerus fractures remains a source of debate and research within orthopaedics. With the advent of locking compression plate technology, open reduction internal fixation (ORIF) of these injuries has become more popular. This operation can offer excellent outcomes-

\footnotetext{
M. B. Berkes $(\bowtie) \cdot$ M. T. M. Little

Hospital for Special Surgery, 535 East 70th Street,

New York, NY 10021, USA

e-mail: berkesm@hss.edu

M. T. M. Little

e-mail: littlem@hss.edu

D. G. Lorich

Orthopaedic Trauma Service, Hospital for Special Surgery and

Weill Cornell Medical College at New York Presbyterian Hospital,

520 East 70th St, Starr Pavilion, 2nd Floor,

New York, NY 10021, USA

e-mail: dg19002@nyp.org
}

not, however, without respect for anatomy and a critical appraisal of the injury pattern, as well as patient- and surgeon-related variables. This article will review the pertinent classic and more recent literature regarding all phases of proximal humerus fracture evaluation and osteosynthesis, as well as the senior author's preferred technique.

\section{Workup and classification}

The appropriate treatment of proximal humerus fractures is predicated on the ability of the treating physician to identify the injury in a timely and accurate fashion and have a complete and thorough understanding of the fracture pattern. Aside from a targeted history and physical exam, imaging remains the mainstay of diagnosis. A plain radiograph trauma series of the shoulder, including an AP, scapular Y, and axillary lateral, is an appropriate first and, possibly, definitive imaging of these images [1]. X-rays provide an overall assessment of alignment, comminution, and bone quality and a basic understanding of the fracture planes involved within the articular segment [2]. Whenever a more detailed understanding of the fracture pattern is required, CT scan provides an excellent means of fracture evaluation. Several studies have demonstrated the benefits of CT scan, as compared with plain radiographs alone. Bahrs et al. demonstrated that CT allowed significantly better assessment of relevant structures, as compared with plain radiographs, even when independent of fracture pattern [3]. Other studies have also demonstrated the benefits of CT scan when used as an adjunct to plain x-rays. This is particularly true for 3-D imaging [4-7]. The utility of MRI is not well described in the setting of acute proximal humerus fracture evaluation but has been described. Voigt compared the use of MRI versus CT and XR in the evaluation of 30 proximal humerus fractures. In their series, MRI was able to accurately demonstrate the bone fracture pattern in all cases 
and also demonstrate rotator cuff tears and medial periosteal hinges that would have been otherwise unrecognized [8]. While expensive and time consuming, this imaging modality certainly holds value and should be considered, especially if soft tissue injury is suspected.

The two most common classification systems for proximal humerus fractures are the Neer and AO classification systems. The Neer classification is based upon the number of fracture "parts," depending on the presence of a fracture plane and sufficient displacement [9]. While its use is ubiquitous, multiple studies have shown that the Neer classification yields low inter- and intraobserver reliability. This is true when plain radiographs are used [7, 10-14], as well as multiplanar CT imaging $[15,16]$. Studies examining the influence of 3-D CT have failed to conclusively demonstrate that this enhances inter-/intraobserver reliability with regard to the Neer classification $[6,17,18]$. Despite the shortcomings of this classification, it serves as a comprehensive classification system, with very few fractures not fitting into this scheme[19]. Additionally, it does carry some prognostic significance, since historically, three- and four-part fractures are more likely to suffer complications and have worse outcomes, as compared with less complex fracture patterns $[20 \cdot, 21-23]$.

The AO classification suffers from many of the shortcomings as the Neer classification. It has been shown to have poor inter-/intraobserver agreement [7, 10, 12-14, 24]. Also, unlike the Neer classification, the AO classification is rather cumbersome and difficult to use in clinical situations.

When evaluating proximal humerus fractures, one should look at a variety of different factors in order to accurately assess the injury and make an appropriate plan for treatment. While the Neer classification is popular, easy to use, and helpful, consideration of other factors, rather than just a simple characterization of "parts," is necessary. For instance, one should make note of bone quality. Hepp, in 2009 , reported that the cortical index of the humerus correlates with risk of fracture, thus serving as a marker for poor bone quality [2]. The condition of the calcar should also be noted. Restoration of the medial calcar is critical for ensuring a successful patient outcome, and this can be complicated by gross displacement or comminution of the calcar $[25,26]$. Some authors even place such importance on this anatomic feature that they consider the calcar the "5th fragment" [27]. Lastly, the pattern of coronal deformity should be noted. Studies have shown that fractures with initial displacement into varus tend to have worse clinical outcomes and higher complication rates if operated upon $[20 \bullet, 21,23,28]$. Thus, when evaluating a proximal humerus fracture and deciding upon appropriate treatment, start with plain radiographs with a low threshold to obtain CT imaging. Orthopaedists should take note of not only the parts involved, but also the condition of the calcar, the level of bone quality, and initial displacement pattern in the coronal plane.

\section{Indications}

Indications for ORIF of proximal humerus fractures are somewhat controversial. Certainly it may be the majority of these fractures can be managed nonoperatively, but a certain percentage should be treated by means of an operation. There are many factors to consider on an individual basis when deciding whether internal fixation should be undertaken, including patient age, functional status, physicosocial factors, ability to tolerate postoperative rehabilitation, fracture pattern, displacement, associated injuries, remaining vascular supply to the articular fragment, and bone quality.

Reported relative indications for proximal humerus fracture ORIF include, but are not limited to, the following: displacement of tuberosities greater than $5 \mathrm{~mm}$, marginal articular fracture with greater than $2 \mathrm{~mm}$ of displacement, greater than $100 \%$ displacement of the surgical neck, or a sufficiently large varus or valgus deformity $[29,30]$. Whether or not internal fixation can be pursued, rather than prosthetic replacement, is dependent upon whether the fracture is reconstructable and whether the blood supply is intact. Fractures that involve a significant head split or multiple articular fragments are generally considered unreconstructable and should be treated with arthroplasty. The issue of remaining blood supply and the ability to predict avascular necrosis (AVN) is incompletely understood. In general, fractures with no remaining soft tissue attachments to the articular segment, particularly in the setting of dislocation, are considered avascular, and arthroplasty has been recommended for these cases in the literature [31]. Hertel reported that calcar length less than $8 \mathrm{~mm}$, disruption of the medial hinge, and more complex fracture patterns were more predictive of future AVN [32]. However, these features cannot absolutely allow the surgeon to predict AVN. Nevaiser et al. found, in their series of 34 patients treated with ORIF, that the length of the posteromedial hinge was not predictive of AVN $[33 \bullet \bullet, 34 \cdot$. Also, using tetracycline labeling techniques, Crosby et al. demonstrated that perfusion to the head can be maintained in more complex three- and four-part fractures [35]. Additionally, Bastian et al. reported that even in cases where ischemia of the head was present at the time of operation, AVN may not occur at long-term follow-up [36]. In this study, 10 humeral heads did not show signs of perfusion intraoperatively. They were treated with internal fixation, and 8 of the 10 did not show any signs of AVN, with subchondral collapse at an average of 5 years followup. This would suggest that revascularization of the head is possible in situations where reduction and stable fixation can be achieved. 
Author's indications for open reduction internal fixation

ORIF should be undertaken if the patient can tolerate the necessary postoperative physical therapy regimen and has sufficient displacement of the tuberosities, articular surface, or surgical neck, as outlined above. Osteosynthesis is pursued even in the presence of risk factors for AVN already mentioned if an adequate reduction and stable fixation can be achieved, in an effort to preserve the native anatomy and provide the best function possible, particularly in younger patients.

\section{Anatomy and surgical approach}

Successful osteosynthesis of proximal humerus fractures by definition requires fracture healing, which is necessarily dependent upon adequate blood supply to the affected fragments. This requires a precise understanding of the vascular anatomy of the proximal humerus. One of the first comprehensive descriptions of the blood supply to the proximal humerus was performed by Gerber et al. [37]. This was a cadaver study performed by injecting latex and studying the vascular contributions to the proximal humerus. In this study, all specimens were perfused by the anterolateral ascending branch of the anterior circumflex artery, which runs parallel to the lateral aspect of the biceps tendon and enters the bone at the proximal end of the intertubercular groove. They also found that only the posterior portion of the greater tuberostiy and the posteroinferior portion of the head was supplied by the posterior humeral circumflex artery. A more recent study by Hettrich et al. suggests that the posterior humeral circumflex artery plays a more important role than previously recognized [38••]. In this cadaver study, the relative contributions of the anterior and posterior humeral circumflex arteries to the overall blood supply of the humeral head was quantified by means of gadolinium uptake on MRI scans. The posterior humeral circumflex artery was found to be the dominant artery, providing, on average, $64 \%$ of the blood supply to the humeral head overall. This finding may help explain why AVN does not necessarily occur despite disruption of the anterior ascending branch, such as in three- or four-part fractures [35]. Surgeons should regard both as clinically important and focus on preserving these vascular structures during the surgical approach, reduction, and fixation.

For open reduction, there are two widely popular approaches available to the surgeon: the deltopectoral and anterolateral acromial, or "deltoid-splitting," approach [39•, 40]. The deltopectoral approach is an attractive option because it follows an internervous plane and does not require dissection of the axillary nerve. However, it provides limited access to the posterior portion of the greater tuberosity and makes placement of a laterally based plate cumbersome. Additionally, the exposure places the anterolateral ascending branch of the anterior humeral circumflex artery at risk during the dissection [41]. The anterolateral acromial, approach is an attractive alternative for proximal humerus osteosynthesis since it avoids many of the pitfalls associated with the deltopectoral approach. It provides direct visualization of the lateral aspect of the humeral shaft for plate placement. Good access to the posterior aspect of the greater tuberosity can be obtained. It respects the vascular supply to the humeral head [42-44]. Additionally, this approach can be performed in a minimally invasive fashion, in an attempt to disrupt as little soft tissue as possible $[45,46]$. Also, this approach can be converted to an extensile lateral approach to the entire humerus if necessary, which requires protection of both the axillary and radial nerves [47]. Drawbacks of the anterolateral approach include the lack of familiarity with gaining inability intra-articular access, should conversion to arthroplasty be required, and concerns regarding injury to the axillary nerve. Certainly, care must be taken to avoid injury to the axillary nerve. However, the nerve is reliably identified $3.5 \mathrm{~cm}$ distal to the tip of the greater tuberosity and with careful handline, nerve injury is rare [48]. Also, damage to the deltoid insertion on the humerus can be avoided with the use of helical plating techniques [49]. Wu et al. performed a retrospective study comparing the anterolateral approach with the deltopectoral approach for proximal humerus fracture treatment and found no difference with regard to operative time, blood loss, hospital stay, fracture reduction, loss of reduction, time to union, Constant-Murley shoulder outcome (Constant) scores, or Disability of Arm, Shoulder, and Hand (DASH) scores [50 ${ }^{\bullet}$. Only three cases of AVN were reported, and all occurred in the deltopectoral approach group. In conclusion, the surgical approach of choice lies at the discretion of the treating orthopaedist. When making this decision, one should consider the fracture pattern and displacement, possible necessity for arthroplasty, surgical experience, and skill; both approaches are suitable when performed appropriately.

\section{Authors preferred technique: Surgical approach}

The senior author favors the anterolateral approach for ORIF of proximal humerus fractures, both in the acute setting and in cases of nonunion [40]. The incision is centered between the anterior and middle heads of the deltoid, with the proximal extent of the incision approximately one finger breadth proximal to the anterolateral border of the acromion [39•]. This incision is extended as far distal as necessary to adequately expose the fracture and facilitate plate placement, while aiming toward the lateral epicondyle of the humerus. Proximally, the interval between the anterior and middle heads of the deltoid is entered using sharp dissection. The axillary nerve is identified during this 
portion of the dissection, which should be approximately $6 \mathrm{~cm}$ distal to the acromion. The subacromial bursa is incised proximal to the nerve to expose the rotator cuff, while blunt dissection is utilized just distal to the axillary nerve to identify the proximal portion of the humeral diaphysis. The axillary nerve need not be completely skeletonized during the dissection; it needs only to be identified and protected (Fig. 1). At this point, fracture reduction can commence, which can be facilitated by the placement of tagging sutures within the rotator cuff tendons to help gain control of tuberosity fragments. These tagging sutures through the rotator cuff can be secured to the plate at the conclusion of the procedure to assist in fixation of the tuberosity fragments.

\section{Fixation and biomechanics}

The goal of proximal humerus fixation is anatomic reduction of all fracture fragments, reestablishment of the native neck shaft angle (between $130^{\circ}$ and $140^{\circ}$ ), restoration of the medial calcar, and stable fixation, which will allow early motion and return to function. The ability of a surgeon to fulfill all of these goals is based on the type of fracture, amount of fracture displacement, bone quality, and patient compliance. ORIF of proximal humerus fractures has increased in frequency with the development of locking plate and angular stable technology. Locking plate technology allows load to be evenly distributed throughout the length of the plate, countering the impact of the humeral head's poor bone quality on plate stability and limiting the risk of screw pull-out and fixation failure.

\section{Calcar reduction/medial column reconstruction}

Care must be taken with the provisional reduction of the major fracture fragments to reduce the calcar and, thus, restore the medial column (Fig. 2). Gardner et al. showed

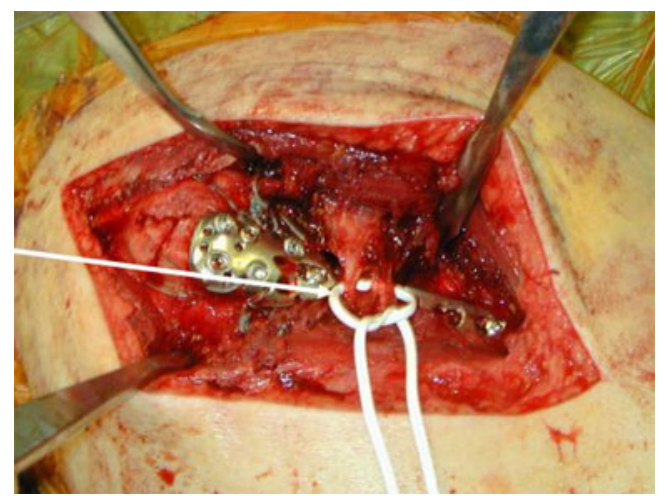

Fig. 1 Intraoperative photographs demonstrating the anterolateral approach to the proximal humerus. Note the location of the axillary nerve, approximately $35 \mathrm{~mm}$ distal to the top of the greater tuberosity and $60-66 \mathrm{~mm}$ distal to the undersurface of the acromion

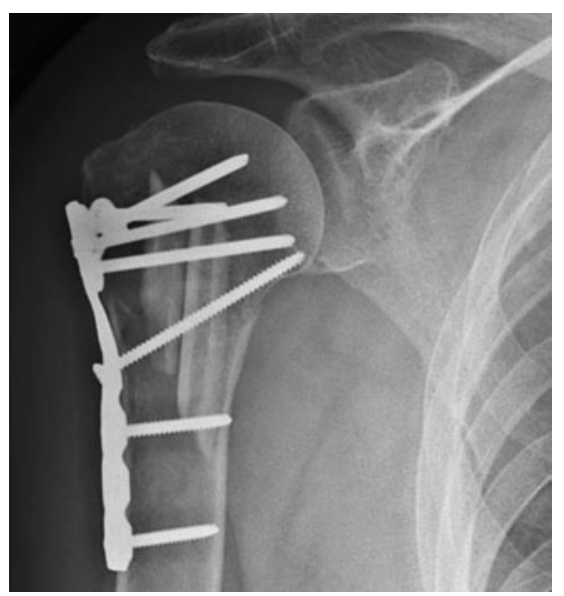

Fig. 2 Postoperative AP radiograph of the shoulder demonstrating excellent reduction of a proximal humerus fracture with locking plate fixation and endosteal augmentation using fibula allograft. Medial support has been achieved through calcar augmentation with allograft fibula and an inferomedially placed screw

that restoration of the medial column, whether through screw fixation or intramedullary fibular strut augmentation, led to a significant improvement in maintenance of humeral head height and fracture reduction, when compared with those without medial support $[25,51 \cdot, 52]$. Biomechanical studies have shown that medial support increases load to failure, axial stiffness, and shear stiffness, as well as torsional stiffness of proximal humerus fixation constructs $[53 \cdot \bullet, 54]$.

Calcar reduction and medial column support is facilitated through numerous methods. Fibular strut augmentation will be discussed in another section. Fractures with minimal calcar comminution allow for anatomic reduction of the calcar and insertion of an appropriately placed calcar screw in the subchondral bone of the inferomedial portion of the humeral head. The positioning of the holes for the calcar screw should be examined carefully prior to plate positioning for fracture fixation. Fractures with significant medial comminution at presentation may require medialization and impaction of the humeral head into the shaft to better restore medial column support. These fractures also benefit from appropriately placed calcar screws through the inferomedial humeral head. Erhardt et al. (2012) showed that placement of an infermomedial/calcar screw increased the constructs resistance to screw perforation and construct failure, when compared with those without an appropriately placed calcar screw [53・•]. Once adequate calcar reduction is achieved, attention can then be turned to plate choice and placement.

Plate choice/plate position

The advent of locking plate technology has increased the frequency of ORIF of proximal humerus fractures. The humeral head consists of a large area of trabecular bone 
with minimal structural support for gaining purchase of screws. Cortical screw fixation is limited in areas without subchondral bone for adequate purchase, and the presence of the articular surface precludes bicortical screw placement. The use of a combination of divergent and convergent screws through an appropriately placed locking plate has improved pull-out strength by distributing forces throughout the plate and depending on the screw-plate interface, rather than the plate-bone interface. Studies have shown no difference in construct strength or functional outcomes when comparing polyaxial and monoaxial locking plates [55-57].

Plate positioning is crucial to both fixation strategies and postoperative motion. As was mentioned previously, following reduction and provisional fixation of the fracture, the plate can be provisionally fixed at the oblong shaft screw. The plate contour should match that of the reduced and stabilized fracture. If possible, the shoulder should be gently abducted to confirm that the proximal aspect of the plate does not impinge on the acromion or limit motion of the extremity. The plate should be positioned $10-15 \mathrm{~mm}$ distal to the greater tuberosity to limit the risk of impingement while providing adequate room for fixation of the humeral head. Erhardt et al. showed that five humeral head screws provided increased resistance to failure and pull-out strength, when compared with three humeral head screws [53••]. Lastly, biomechanical data has demonstrated that screws should be placed into the subchondral bone of the humeral head, less than 5 millimeters from the articular surface, to provide the best fixation [58].

Locking screws are placed in the head in a divergent and convergent fashion to ensure increased purchase, while cortical screws can be placed in the humeral shaft when a patient's bone quality permits. Cortical screw placement will allow the surgeon to utilize the plate as a reduction tool in fractures where provisional stability may be limited initially. Screw position should be checked in multiple views, and the proximal humerus should be taken through a full arc of motion to confirm stability and confirm that the humeral head has not been perforated. Following fixation, the tagging sutures utilized for manipulation of the fracture fragments can be passed through holes in the locking plate for reinforcement of the fixation [59].

\section{Fixation augmentation}

While locking plate utilization has increased significantly, complications and poor outcomes remain due to the poor quality of humeral head bone. Fixation augmentation with allograft fibular struts has become a more popular means of countering the low bone density of the humeral head and its impact on maintenance of fixation. The technique of fibula placement was described by Gardner, Lorich, and Helfet et al. in 2008 [26]. The fibula is introduced in the proximal humerus via the preexisting lateral fracture line in an antegrade fashion (Fig. 3). In general, the fibula acts a second, more medial point of fixation for screws placed through the locking plate, enhancing the strength of fixation. In varus fractures, the fibular strut can be placed medial to reestablish the medial column and provide increased structural support and a strong point of fixation for calcar screws and screws placed through the humeral head. In valgus-impacted fractures, the fibula can be placed in a more lateral position to fill the void left by the disimpacted head fragment. The fibula acts as a reduction device that provides provisional stability and allows the surgeon to elevate and translate the major fracture fragments with precision, while limiting the need for multiple surgical assistants to maintain fracture reduction (Fig. 4). This stability improves the surgeon's ability to place the plate appropriately without loss of reduction.

Biomechanical studies have shown increased resistance to collapse, increased maximal failure load, and improved construct stiffness with fibular strut augmentation [51•, 60-62]. Fibular strut augmentation has been shown to decrease cyclic motion at the fracture site by five times and lower fragment motion by two times, as well as decrease plastic deformation by two times, in a study by Osterhoff et al. [51•]. These findings were confirmed in clinical studies by Hettrich et al. (2012) showing excellent maintenance of reduction and postoperative outcomes in patients treated with fibular strut augmentation of fixation [63•]. Fibular strut augmentation should be considered in geriatric patients, as well as those patients with poorer bone quality or more comminuted fractures, to improve fixation.

\section{Outcomes and complications}

Complications of proximal humeral fixation remain prevalent despite the increased use of angular stable plates. They can be attributed to fracture reduction, plate position, and preoperative displacement. Rates of postoperative complications

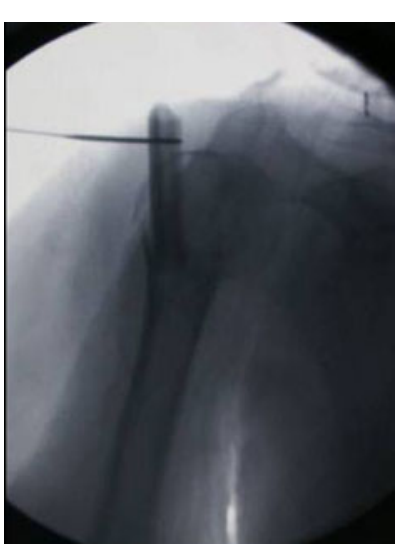

Fig. 3 Placement of the allograft fibula through the fracture site and into the metaphyseal bone 
Fig. 4 Allograft fibula placement. The fibula is medialized with a push screw, placed through the plate. The medialization of the fibula helps assist in the reduction and restoration of the calcar. Locking screws are also placed through the fibula to hold its position and prevent postoperative loss of reduction

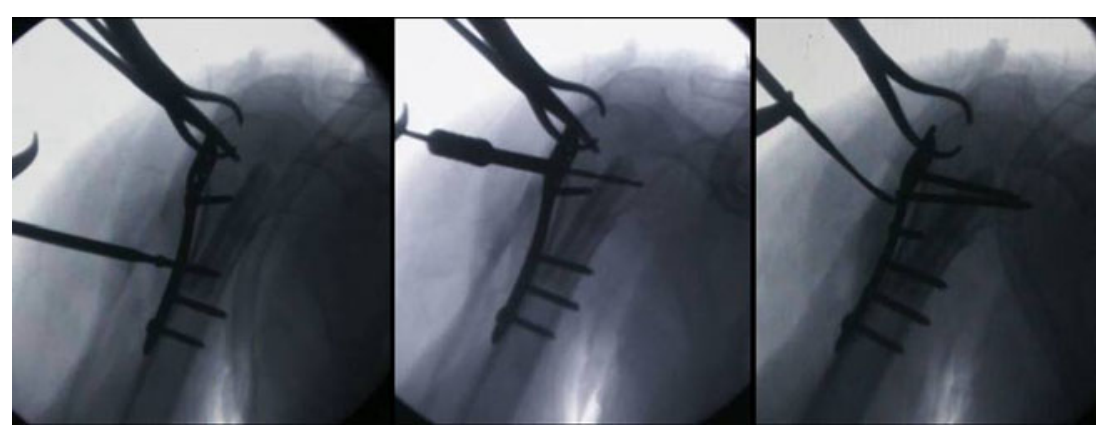

range from $9.7 \%$ to $39 \%$ of patients $[64,65]$. Some less common complications include subacromial impingement due to plate positioning, adhesive capsulitis, and postoperative superficial/deep infections. These complications were reported at decreased frequency, but physicians should be aware of their presence. Adhesive capsulitis is often attributed to delayed initiation of physical therapy following fixation. The numerous complications have contributed to reoperation rates as high as $29 \%$ in some studies [66].

Screw perforation of the humeral head is the most common complication, with reported rates of up to $17 \%$ in fractures treated without fixation augmentation [67]. Konigshasen et al. reported that nearly half ( $42 \%$ ) of their study's complications were attributed to screw perforation of the humeral head [68]. Postoperative radiographs should be monitored periodically for signs of perforation at each visit. The shoulder should be taken through an arc of motion for evaluation of crepitus or catching. Screws should be removed in a timely manner to limit injury to the articular surface of the glenoid if fracture fixation is not negatively impacted by screw removal. Neviaser et al. showed a significant improvement in the rate of screw perforation with augmentation of fixation with a fibular strut allograft [33••]. There were zero cases of screw perforation in the series, which is likely attributed to their low rate of loss of humeral height, change in neck shaft angle, and loss of reduction.

Varus malreduction is another commonly reported complication of proximal humerus fixation [69]. Patients' presenting with initial varus coronal displacement portends poorer postoperative coronal alignment. Solberg et al., in 2009 , reported that $70 \%$ of their patients with preoperative varus alignment were still in at least $5^{\circ}$ of varus after undergoing fixation [20•]. This cohort had worse functional outcomes at follow-up and was associated with a complication rate of $79 \%$. Early evidence of progression of varus malreduction should be treated with revision ORIF to limit the risk of further fixation failure and poor outcomes.

AVN of the humeral head was previously considered one of the risk factors of operative treatment of three- and four-part proximal humerus fractures. Rates of AVN have been reported from $0 \%$ to $30 \%[34 \bullet, 65,70-74]$ (Neviaser, Hettrich, Dines, et al., 2011; Schliemann et al., 2012; Schulte, Matteini, \&
Neviaser, 2011). The high rate of AVN was previously attributed to the perception that the anterior humeral circumflex artery was the major contributing vessel to the humeral head. While the rate appears high, it would not take into account the frequency with which the anterior humeral circumflex was disrupted, whether by injury or surgical fixation. Hettrich et al. showed that the posterior circumflex artery was the major contributing vessel to the humeral head, supporting the theory that these injuries should be associated with a low rate of AVN [38••]. Radiographic evidence of AVN (subchondral collapse, humeral head segmentation) should be followed closely with postoperative imaging, but surgical intervention may be delayed in asymptomatic patients with good functional outcomes, limited functional requirements, and intact hardware, with no signs of humeral head perforation. Conversion to hemiarthroplasty or total arthroplasty should be considered in patients with early development of AVN and limited function.

Outcomes of proximal humerus fixation vary widely and are highly dependent on a surgeon's treatment philosophy and preoperative presentation. The Constant shoulder outcome score, the UCLA shoulder score, and the DASH score are common functional outcome scores utilized for these injuries. The Constant score is an objective method of evaluating patient outcomes on the basis of range of motion, strength, pain, and activity level. The UCLA score is similarly weighted but also includes patient satisfaction and function. The DASH is a more global assessment of the upper extremity and the patients overall well-being. Constant scores have ranged from 61 to 80 ( $\max 100)$ with angular stable constructs [20•, 21, 75], but significant variability remains. Solberg et al. (2009) and Sudkamnp et al. (2011) reported poorer Constant scores in their patients presenting with varus preoperative coronal displacement, as compared with their valgus-impacted patients $[20 \bullet, 76]$. In contrast, Hettrich et al. reported Constant scores of 87 in their cohort of patients treated with fibular strut augmentation of the angular stable [63•]. The fixation strategy was associated with a low rate of complications and low rate of loss of reduction. DASH and UCLA scores exhibit similar variability based on surgeon's treatment strategy. Uniformly, patients with anatomic fracture reduction and reestablishment of the medial calcar are associated with significantly better DASH, UCLA, and Constant scores [25, 63•, 69, 74, 77]. 


\section{Conclusion}

The treatment of proximal humerus fractures continues to evolve with fixation method and treatment protocols. With the growing numbers of geriatric patients, the frequency of these injuries will only increase. Research has shown that despite the myriad of treatment methods, preoperative risk factors, and augmentation, anatomic reduction and reestablishment of the medial column remain crucial to maintenance of reduction and good functional outcomes.

Key learning points

- Shoulder series is essential for appropriate treatment of proximal humerus fractures (AP, scapular Y, axillary).

- Computed tomography provides detailed evaluation of fracture classification and may direct treatment.

- Fractures may be classified by the Neer or AO classification systems, both of which have a variety of shortcomings. Neer 3 and 4 fractures have historically been associated with poorer outcomes.

- Calcar stability and preoperative varus coronal alignment are considered additional prognostic factors when determining the appropriate treatment strategy for these injuries.

- Relative surgical indications are displacement of tuberosities greater than $5 \mathrm{~mm}$, marginal articular fracture with greater than $2 \mathrm{~mm}$ of displacement, greater than $100 \%$ displacement of the surgical neck, or a sufficiently large varus or valgus deformity, and ability to complete postoperative physical therapy regimen.

- The two most common surgical approaches to fixation are the deltopectoral and the anterolateral/deltoid-splitting approaches.

- Advantages of the anterolateral approach include exposure of the lateral aspect of the proximal humerus for plate placement, ability to convert to extensile approach. Disadvantages of this approach include minimal visualization of the articular surface and inability to convert to hemi-arthroplasty.

- Advantages of the deltopectoral approach include internervous plane for dissection and avoidance of the axillary nerve. Disadvantages include limited exposure of the posterior greater tuberosity and cumbersome plate placement.

- Adequate reduction of all fracture fragments is crucial to anatomic fixation. Tagging sutures may be utilized to counter deforming forces of the muscular attachments.

- Locking plate technology has improved fixation of these fractures and decreased the risk of screw pull-out from the humeral head.

- Reestablishment of the medial column though fibular augmentation and/or appropriate calcar screw placement improves maintenance of reduction.
- Complications of fracture fixation remain despite the use of locking plates and plate augmentation. Screw penetration of the humeral head, AVN, and varus malreduction should be monitored closely throughout follow-up.

- Regardless of the method of fixation utilized, functional outcomes are dependent on anatomic reduction, restoration of the medial column, and a patient's ability to participate in their postoperative rehabilitation course.

Disclosure No potential conflicts of interest relevant to this article were reported.

\section{References}

Papers of particular interest, published recently, have been highlighted as:

- Of importance

•. Of major importance

1. Rockwood CA, Green DP, Bucholz RW, Heckman JD. Rockwood and green's fractures in adults. 5th ed. Philadelphia: Lippincott Williams \& Wilkins; 2001.

2. Hepp P, Theopold J, Osterhoff G, et al. Bone quality measured by the radiogrammetric parameter "cortical index" and reoperations after locking plate osteosynthesis in patients sustaining proximal humerus fractures. Arch Orthop Trauma Surg. 2009;129(9):1251-9.

3. Bahrs C, Rolauffs B, Sudkamp NP, et al. Indications for computed tomography (CT-) diagnostics in proximal humeral fractures: A comparative study of plain radiography and computed tomography. BMC Musculoskelet Disord. 2009;10:33.

4. Brunner A, Honigmann P, Treumann T, Babst R. The impact of stereo-visualisation of three-dimensional CT datasets on the interand intraobserver reliability of the AO/OTA and Neer classifications in the assessment of fractures of the proximal humerus. $\mathrm{J}$ Bone Joint Surg Br. 2009;91(6):766-71.

5. Edelson G, Saffuri H, Obid E, Vigder F. The three-dimensional anatomy of proximal humeral fractures. J Should Elb Surg Am Should Elbow Surg [Et Al]. 2009;18(4):535-44.

6. Foroohar A, Tosti R, Richmond JM, et al. Classification and treatment of proximal humerus fractures: inter-observer reliability and agreement across imaging modalities and experience. J Orthop Surg Res. 2011;6:38.

7. Gumina S, Giannicola G, Albino P, et al. Comparison between two classifications of humeral head fractures: Neer and AO-ASIF. Acta Orthop Belg. 2011;77(6):751-7.

8. Voigt C, Ewig M, Vosshenrich R, Lill H. Value of MRI in preoperative diagnostics of proximal humeral fractures compared to $\mathrm{CT}$ and conventional radiography. [Wertigkeit der MRT in der praoperativen Diagnostik proximaler Humerusfrakturen vs. CT und konventionelles Rontgen]. Der Unfallchirurg. 2010;113(5):378-85.

9. Neer 2nd CS. Displaced proximal humeral fractures. I. Classification and evaluation. J Bone Joint Surg Br Vol. 1970;52 (6):1077-89.

10. Brien H, Noftall F, MacMaster S, et al. Neer's classification system: a critical appraisal. J Trauma. 1995;38(2):257-60. 
11. Brorson S, Bagger J, Sylvest A, Hrobjartsson A. Improved interobserver variation after training of doctors in the neer system. A randomised trial. J Bone Jt Surg Br Vol. 2002;84(7):950-4.

12. Kristiansen B, Andersen UL, Olsen CA, Varmarken JE. The neer classification of fractures of the proximal humerus. an assessment of interobserver variation. Skelet Radiol. 1988;17(6):420-2.

13. Sidor ML, Zuckerman JD, Lyon T, et al. The neer classification system for proximal humeral fractures. an assessment of interobserver reliability and intraobserver reproducibility. J Bone Joint Surg Am Vol. 1993;75(12):1745-50.

14. Siebenrock KA, Gerber C. The reproducibility of classification of fractures of the proximal end of the humerus. J Bone Joint Surg Am Vol. 1993;75(12):1751-5.

15. Bernstein J, Adler LM, Blank JE, et al. Evaluation of the neer system of classification of proximal humeral fractures with computerized tomographic scans and plain radiographs. J Bone Joint Surg Am Vol. 1996;78(9):1371-5.

16. Sjoden GO, Movin T, Guntner P, et al. Poor reproducibility of classification of proximal humeral fractures. additional CT of minor value. Acta Orthop Scand. 1997;68(3):239-42.

17. Brunner A, Honigmann P, Treumann T, Babst R. The impact of stereo-visualisation of three-dimensional CT datasets on the interand intraobserver reliability of the AO/OTA and neer classifications in the assessment of fractures of the proximal humerus. $J$ Bone Joint Surg Br Vol. 2009;91(6):766-71.

18. Sjoden GO, Movin T, Aspelin P, et al. 3D-radiographic analysis does not improve the neer and AO classifications of proximal humeral fractures. Acta Orthop Scand. 1999;70(4):325-8.

19. Tamai K, Ishige N, Kuroda S, et al. Four-segment classification of proximal humeral fractures revisited: A multicenter study on 509 cases. J Should Elb Surg Am Should Elbow Surg [Et Al]. 2009;18 (6):845-50.

20. - Solberg BD, Moon CN, Franco DP, Paiement GD. Locked plating of 3- and 4-part proximal humerus fractures in older patients: the effect of initial fracture pattern on outcome. J Orthop Trauma. 2009;23(2):113-9. Comparison of two cohorts of fractures presenting with varus (24) or valgus (27) preoperative coronal displacement in three- and four-part fractures. Constant scores were significantly higher for the valgus group (71 vs. 63) at final follow-up. Overall complication rate, including AVN, screw perforation, loss of fixation, and displacement, was $79 \%$ in the varus group, as compared with $19 \%$ in the valgus group. Initial fracture pattern is prognostic for postoperative outcomes.

21. Solberg BD, Moon CN, Franco DP, Paiement GD. Surgical treatment of three and four-part proximal humeral fractures. J Bone Joint Surg Am Vol. 2009;91(7):1689-97.

22. Spross C, Platz A, Rufibach K, et al. The PHILOS plate for proximal humeral fractures-risk factors for complications at one year. J Trauma Acute Care Surg. 2012;72(3):783-92.

23. Yang H, Li Z, Zhou F, et al. A prospective clinical study of proximal humerus fractures treated with a locking proximal humerus plate. J Orthop Trauma. 2011;25(1):11-7.

24. Brorson S, Bagger J, Sylvest A, Hrobjartsson A. Low agreement among 24 doctors using the neer-classification; only moderate agreement on displacement, even between specialists. Int Orthop. 2002;26(5):271-3.

25. Gardner MJ, Weil Y, Barker JU, et al. The importance of medial support in locked plating of proximal humerus fractures. J Orthop Trauma. 2007;21(3):185-91.

26. Gardner MJ, Boraiah S, Helfet DL, Lorich DG. Indirect medial reduction and strut support of proximal humerus fractures using an endosteal implant. J Orthop Trauma. 2008;22(3):195-200.

27. Russo R, Cautiero F, Della Rotonda G. The classification of complex 4-part humeral fractures revisited: the missing fifth fragment and indications for surgery. Musculoskelet Surg. 2012;96 Suppl 1:S13-9.
28. Sudkamp NP, Audige L, Lambert S, Hertel R, et al. Path analysis of factors for functional outcome at one year in 463 proximal humeral fractures. J Should Elb Surg Am Should Elbow Surg [Et Al]. 2011;20(8):1207-16.

29. Guy P, Slobogean GP, McCormack RG. Treatment preferences for displaced three- and four-part proximal humerus fractures. J Orthop Trauma. 2010;24(4):250-4.

30. Robinson CM, Amin AK, Godley KC, et al. Modern perspectives of open reduction and plate fixation of proximal humerus fractures. J Orthop Trauma. 2011;25(10):618-29.

31. Robinson CM, Khan LA, Akhtar MA. Treatment of anterior fracture-dislocations of the proximal humerus by open reduction and internal fixation. J Bone Joint Surg Am Vol. 2006;88 (4):502-8.

32. Hertel R, Hempfing A, Stiehler M, Leunig M. Predictors of humeral head ischemia after intracapsular fracture of the proximal humerus. J Should Elb Surg Am Should Elbow Surg [Et Al]. 2004;13(4):427-33.

33. •- Neviaser AS, Hettrich CM, Beamer BS, et al. Endosteal strut augment reduces complications associated with proximal humeral locking plates. Clin Orthop Relat Res. 2011;469(12):3300-6. This is one of the first clinical studies to examine the clinical effects and complications associated with endosteal strut augmentation of proximal humeral locking plates. No episodes of intra-articular screw penetration or cutout were reported in this series of 38 patients. One episode of partial AVN and one patient with loss of reduction were reported. The mean DASH score was 15 , and the mean Constant score was 87.

34. - Neviaser AS, Hettrich CM, Dines JS, Lorich DG. Rate of avascular necrosis following proximal humerus fractures treated with a lateral locking plate and endosteal implant. Arch Orthop Trauma Surg. 2011;131(12):1617-22. Evaluation of rate of AVN following proximal humerus fractures treated with endosteal augmentation and proximal humeral locking plates. Thirty-four patients were followed up at a mean of 66 weeks. There was a $0 \%$ rate of complete AVN and a $2.8 \%$ rate of partial AVN without collapse. Length of posteromedial hinge was not found to be predictive of AVN in this patient series.

35. Crosby LA, Finnan RP, Anderson CG, et al. Tetracycline labeling as a measure of humeral head viability after 3- or 4-part proximal humerus fracture. J Should Elb Surg Am Should Elbow Surg [Et Al]. 2009;18(6):851-8.

36. Bastian JD, Hertel R. Initial post-fracture humeral head ischemia does not predict development of necrosis. J Should Elb Surg Am Should Elbow Surg [Et Al]. 2008;17(1):2-8.

37. Gerber C, Schneeberger AG, Vinh TS. The arterial vascularization of the humeral head. an anatomical study. J Bone Joint Surg Am Vol. 1990;72(10):1486-94.

38. • Hettrich CM, Boraiah S, Dyke JP, et al. Quantitative assessment of the vascularity of the proximal part of the humerus. J Bone Joint Surg Am Vol. 2010;92(4):943-8. This anatomic cadaver study was performed to reevaluate the vascular supply to the proximal humerus with the assistance of latex injection, as well as MRI imaging with the assistance of gadolinium. The posterior humeral circumflex artery displayed a dominant pattern, supplying approximately $64 \%$ of the humeral head. This lies in contrast to the findings of Gerber et al., who reported the anterior humerual cirumflex as the dominant artery. This finding may help explain why AVN is not seen as frequently as expected in cases in which the anterior humeral circumflex artery is disrupted.

39. • Gardner MJ, Boraiah S, Helfet DL, Lorich DG. The anterolateral acromial approach for fractures of the proximal humerus. J Orthop Trauma. 2008;22(2):132-7. This article provides a detailed description of the anterolateral approach to the proximal humerus. The technique and benefits of the surgical approach are highlighted. 
40. Hettrich CM, Paul O, Neviaser AS, et al. The anterolateral approach to the proximal humerus for nonunions and delayed unions. Int J Should Surg. 2011;5(1):21-5.

41. Gavaskar AS, Muthukumar S, Chowdary N. Biological osteosynthesis of complex proximal humerus fractures: surgical technique and results from a prospective single center trial. Arch Orthop Trauma Surg. 2010;130(5):667-72.

42. Gardner MJ, Griffith MH, Dines JS, Lorich DG. A minimally invasive approach for plate fixation of the proximal humerus. Bull Hosp Jt Dis (New York NY). 2004;62(1-2):18-23.

43. Gardner MJ, Griffith MH, Dines JS, et al. The extended anterolateral acromial approach allows minimally invasive access to the proximal humerus. Clin Orthop Relat Res. 2005;434:123-9.

44. Gardner MJ, Voos JE, Wanich T, et al. Vascular implications of minimally invasive plating of proximal humerus fractures. J Orthop Trauma. 2006;20(9):602-7.

45. Roderer G, Erhardt J, Graf M, et al. Clinical results for minimally invasive locked plating of proximal humerus fractures. J Orthop Trauma. 2010;24(7):400-6.

46. Ruchholtz S, Hauk C, Lewan U, et al. Minimally invasive polyaxial locking plate fixation of proximal humeral fractures: a prospective study. J Traumatol. 2011;71(6):1737-44.

47. Robinson CM, Murray IR. The extended deltoid-splitting approach to the proximal humerus: variations and extensions. J Bone Joint Surg Am Vol. 2011;93(3):387-92.

48. Khan LA, Robinson CM, Will E, Whittaker R. Assessment of axillary nerve function and functional outcome after fixation of complex proximal humeral fractures using the extended deltoidsplitting approach. Injury. 2009;40(2):181-5.

49. Gardner MJ, Griffith MH, Lorich DG. Helical plating of the proximal humerus. Injury. 2005;36(10):1197-200.

50. - Wu $\mathrm{CH}$, Ma $\mathrm{CH}$, Yeh JJ, et al. Locked plating for proximal humeral fractures: differences between the deltopectoral and deltoid-splitting approaches. J Trauma. 2011;71(5):1364-70. Retrospective study comparing outcomes of proximal humerus fracture operative treatment utilizing either the anterolateral or the deltopectoral approach. No statistically significant differences in outcome were found. However, all three cases of AVN were seen in the deltopectoral group.

51. - Osterhoff G, Baumgartner D, Favre P, et al. Medial support by fibula bone graft in angular stable plate fixation of proximal humeral fractures: an in vitro study with synthetic bone. J Should Elb Surg Am Should Elbow Surg [Et Al]. 2011;20(5):740-6. Biomechanical study with synthetic bone fixed with an angular stable locking plate with or without and endosteal fibular strut as a medial support. The models with the endosteal fibular strut had five times lower intercylcic motion, two times lower fragment migration, and two times less residual plastic deformation, as compared with the models without a fibular strut.

52. Osterhoff G, Ossendorf C, Wanner GA, et al. The calcar screw in angular stable plate fixation of proximal humeral fractures-a case study. J Orthop Surg Res. 2011;6:50.

53. • Erhardt JB, Stoffel K, Kampshoff J, et al. The position and number of screws influence screw perforation of the humeral head in modern locking plates: a cadaver study. J Orthop Trauma. 2012;26(10):e188-92. Biomechanical study in cadavers. A key finding was that more screws correlated with increasing load to failure. Additionally, the inferomedial screw (the calcar screw) was found to be biomechanically the most important.

54. Lescheid J, Zdero R, Shah S, et al. The biomechanics of locked plating for repairing proximal humerus fractures with or without medial cortical support. J Trauma. 2010;69(5):1235-42.

55. Ockert B, Braunstein V, Kirchhoff C, et al. Monoaxial versus polyaxial screw insertion in angular stable plate fixation of proximal humeral fractures: Radiographic analysis of a prospective randomized study. J Trauma. 2010;69(6):1545-51.
56. Shi HF, Xiong J, Chen YX, et al. Management of proximal humeral fractures in elderly patients with uni- or polyaxial locking osteosynthesis system. Arch Orthop Trauma Surg. 2011;131(4):541-7.

57. Voigt C, Geisler A, Hepp P, et al. Are polyaxially locked screws advantageous in the plate osteosynthesis of proximal humeral fractures in the elderly? A prospective randomized clinical observational study. J Orthop Trauma. 2011;25(10):596-602.

58. Liew AS, Johnson JA, Patterson SD, et al. Effect of screw placement on fixation in the huemral head. J Shoulder Elbow Surg. 2000;9(5):423-6.

59. Dimakopoulos P, Panagopoulos A, Kasimatis G. Transosseous suture fixation of proximal humeral fractures. Surgical technique. J Bone Joint Surg Am Vol. 2009;91(Suppl 2 Pt 1):8-21.

60. Bae JH, Oh JK, Chon CS, et al. The biomechanical performance of locking plate fixation with intramedullary fibular strut graft augmentation in the treatment of unstable fractures of the proximal humerus. J Bone Joint Surg Am Vol. 2011;93 (7):937-41.

61. Chow RM, Begum F, Beaupre LA, et al. Proximal humeral fracture fixation: locking plate construct $+/$ - intramedullary fibular allograft. J Should Elb Surg. 2012;21(7):894-901.

62. Mathison C, Chaudhary R, Beaupre L, et al. Biomechanical analysis of proximal humeral fixation using locking plate fixation with an intramedullary fibular allograft. Clin Biomech (Bristol, Avon). 2010;25(7):642-6.

63. - Hettrich CM, Neviaser A, Beamer BS, et al. Locked plating of the proximal humerus using an endosteal implant. J Orthop Trauma. 2012;26(4):212-5. Study examining outcomes of geriatric patients treated with ORIF using endosteal fibular strut. In these 27 patients, all older than 70 years, only one case experienced a loss of reduction. No cases experienced screw penetration or AVN. This demonstrates the utility of the endosteal support, even in older individuals with poorer bone quality.

64. Aksu N, Gogus A, Kara AN, Isiklar ZU. Complications encountered in proximal humerus fractures treated with locking plate fixation. Acta Orthop Traumatol Turc. 2010;44(2):89-96.

65. Faraj D, Kooistra BW, Vd Stappen WA, Werre AJ. Results of 131 consecutive operated patients with a displaced proximal humerus fracture: an analysis with more than two years follow-up. Eur J Orthop Surg Traumatol Orthop Traumatol. 2011;21(1):7-12.

66. Hardeman F, Bollars P, Donnelly M, Bellemans J, Nijs S. Predictive factors fro functional outcome and failure in angular stable osteosynthesis of the proximal humerus. Injury. 2012;43(2):153-8.

67. Olerud P, Ahrengart L, Ponzer S, Saving J, Tidermark J. Internal fixation versus nonoperative treatment of displaced 3-part proximal humeral fractures in elderly patients: a randomized controlled trial. J Should Elb Surg Am Should Elbow Surg [Et Al]. 2011;20 (5):747-55.

68. Konigshausen M, Kubler L, Godry H, et al. Clinical outcome and complications using a polyaxial locking plate in the treatment of displaced proximal humerus fractures. A reliable system? Injury. 2012;43(2):223-31.

69. Lee CW, Shin SJ. Prognostic factors for unstable proximal humeral fractures treated with locking-plate fixation. J Should Elb Surg Am Should Elbow Surg [Et Al]. 2009;18(1):83-8.

70. Badman B, Frankle M, Keating C, Henderson L, et al. Results of proximal humeral locked plating with supplemental suture fixation of rotator cuff. J Should Elb Surg Am Should Elbow Surg [Et Al]. 2011;20(4):616-24.

71. Brunner F, Sommer C, Bahrs C, et al. Open reduction and internal fixation of proximal humerus fractures using a proximal humeral locked plate: a prospective multicenter analysis. J Orthop Trauma. 2009;23(3):163-72.

72. Duralde XA, Leddy LR. The results of ORIF of displaced unstable proximal humeral fractures using a locking plate. J Should Elb Surg Am Should Elbow Surg [Et Al]. 2010;19(4):480-8. 
73. Schliemann B, Siemoneit J, Theisen C, et al. Complex fractures of the proximal humerus in the elderly-outcome and complications after locking plate fixation. Musculoskelet Surg. 2012;96 Suppl 1: S3-S11.

74. Schulte LM, Matteini LE, Neviaser RJ. Proximal periarticular locking plates in proximal humeral fractures: functional outcomes. J Should Elb Surg Am Should Elbow Surg [Et Al]. 2011;20 (8):1234-40.

75. Siwach R, Singh R, Rohilla RK, et al. Internal fixation of proximal humeral fractures with locking proximal humeral plate (LPHP) in elderly patients with osteoporosis. J Orthop Traumatol Off J Ital Soc Orthop Traumatol. 2008;9(3):149-53.

76. Sudkamp N, Bayer J, Hepp P, et al. Open reduction and internal fixation of proximal humeral fractures with use of the locking proximal humerus plate. Results of a prospective, multicenter, observational study. J Bone Joint Surg Am Vol. 2009;91(6):1320-8.

77. Zhang L, Zheng J, Wang W, et al. The clinical benefit of medial support screws in locking plating of proximal humerus fractures: a prospective randomized study. Int Orthop. 2011;35 (11):1655-61. 